\title{
Diary
}

\section{2-23 May}

Educational Technology Users' Conference Dundalk, Ireland.

Information: See website - registration and login required. http://ilta.learnonline.ie/login/index.php

\section{3-14 June}

Fourth Inter-Varietal Applied Corpus Studies (IVACS) group International Conference on Applying Corpus Linguistics

Limerick, Ireland

Information: Elaine Vaughan

http://www.mic.ul.ie/ivacs/

31 May-1 June

JALTCALL 2008

Nagoya, Japan

Information: c2008@jaltcall.org

18-19 June

Third International Blended Learning

Conference

Hatfield, UK

Information: Liz Mellor, The Blended Learning

Unit, University of Hertfordshire, College Lane Campus, Hatfield, HERTS AL10 9AB, UK

Tel: +44 (0)1707 281316

4 July

ED-MEDIA 2008 - World Conference on

Educational Multimedia, Hypermedia \&

Telecommunications

Vienna, Austria

Information:info@aace.org

8-9 July

12th International Computer-Assisted

Assessment conference

Loughborough, UK

Information: Sonya Medlock, Loughborough

University Leicestershire LE11 3TU, UK

Tel: +44(0)1509 223736 / Fax: +44(0)1509 223927

http://www.caaconference.com/

\section{2-18 July}

Designing Activities for the 2.0 Language

Classroom

Utrecht, Netherlands

Information: Ton Koenraad

http://www.utrechtsummerschool.nl/index.php?pa ge $=$ courses $\&$ code $=\mathrm{S} 5$
17-18 July

Teaching and Learning through Gaming and Simulation

http://www.nottingham.ac.uk/business/game2008/

Nottingham, UK

Information: Alison Parrett. Tel: +44 (0) 1159514011

\section{5-8 August}

\section{WorldCALL 2008}

Fukuoka, Japan

Information: Prof Mike Levy

http:// www.worldcall.org/

\section{4-28 August}

AILA 2008-the 15th World Congress of

Applied Linguistics

Essen, Germany

Information: Julian Sudhoff, Universität

Duisburg-Essen, Campus Essen, Fachbereich

Geisteswissenschaften, Anglistik,

Universitätsstraße 12, 45117 Essen, Germany http://www.aila2008.org/ / Tel.: +49-201-183-3461

30 August-2 September

Antwerp CALL 2008: XIIIth International

CALL Research Conference

Antwerp, Belgium

Information: Ann Aerts, Conference manager,

LINGUAPOLIS - University of

Antwerp, Prinsstraat 8, 2000 Antwerp, Belgium

Tel: +3232204197 / Fax: +3232204637

http://www.ua.ac.be/main.aspx?c=.CALL2008

\section{3-6 September}

\section{EUROCALL 2008}

Székesfehérvár, Hungary

Information: http://www.asszisztencia.hu/eurocall/

17-19 September

Third European Conference on Technology

Enhanced Learning

Maastricht, Netherlands

Information: http://ectel08.org

18-20 September

7th Annual International Conference of the

European Association of

Languages for Specific Purposes (AELFE)

La Manga, Spain

Information: aelfe2008@um.es

Please note: an up-to-date and expanded version of this Diary may be found at http://www.eurocall-languages.org/resources/calendar.htm 